\section{BRAZIULIAN JOURNAL}

OF MEDICAL AND BIOLOGICAL RESFARCH

www.bjournal.com.br
ISSN 0100-879X

Volume 44 (7) 606-728 July 2011

BIOMEDICAL SCIENCES

AND

CLINICAL INVESTIGATION

Braz J Med Biol Res, July 2011, Volume 44(7) 624-633

doi: 10.1590/S0100-879X2011007500077

Dermatan sulfate reduces monocyte chemoattractant protein 1 and TGF- $\beta$ production, as well as macrophage recruitment and myofibroblast accumulation in mice with unilateral ureteral obstruction

C.L.R. Belmiro, R.G. Gonçalves, E.O. Kozlowski, A.F. Werneck, C.M. Takyia, M. Leite-Jr. and M.S.G. Pavão

The Brazilian Journal of Medical and Biological Research is partially financed by
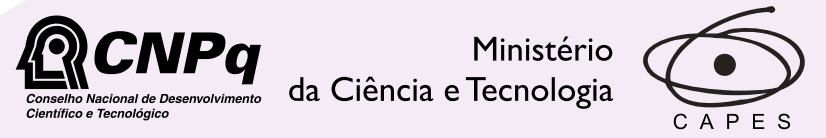

Ministério da Educação
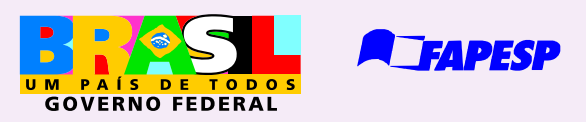

Institutional Sponsors
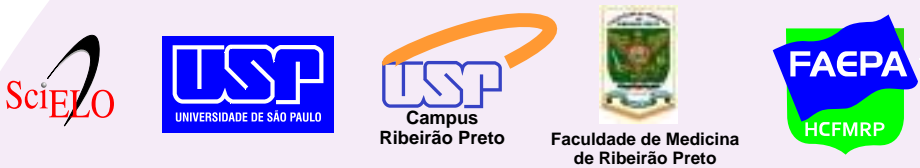


\title{
Dermatan sulfate reduces monocyte chemoattractant protein 1 and TGF- $\beta$ production, as well as macrophage recruitment and myofibroblast accumulation in mice with unilateral ureteral obstruction
}

\author{
C.L.R. Belmiro ${ }^{1 *}$, R.G. Gonçalves ${ }^{2 *}$, E.O. Kozlowski ${ }^{1}$, A.F. Werneck ${ }^{1}$, \\ C.M. Takyia ${ }^{3}$, M. Leite-Jr. ${ }^{2}$ and M.S.G. Pavão ${ }^{1}$ \\ ${ }^{1}$ Laboratório de Bioquímica e Biologia Celular de Glicoconjugados, Programa de Glicobiologia, \\ Instituto de Bioquímica Médica and Hospital Universitário Clementino Fraga Filho, \\ Universidade Federal do Rio de Janeiro, Rio de Janeiro, RJ, Brasil \\ 2Serviço de Nefrologia, Departamento de Clínica Médica, Hospital Universitário Clementino Fraga Filho, \\ Universidade Federal do Rio de Janeiro, Rio de Janeiro, RJ, Brasil ${ }^{3}$ Departamento de Histologia e Embriologia, \\ Instituto de Ciências Biomédicas, \\ Universidade Federal do Rio de Janeiro, Rio de Janeiro, RJ, Brasil
}

\begin{abstract}
Selectins play an essential role in most inflammatory reactions, mediating the initial leukocyte-rolling event on activated endothelium. Heparin and dermatan sulfate (DS) bind and block P- and L-selectin function in vitro. Recently, we reported that subcutaneous administration of DS inhibits colon inflammation in rats by reducing macrophage and T-cell recruitment, and macrophage activation. In the present study, we examined the effect of porcine intestinal mucosa DS on renal inflammation and fibrosis in mice after unilateral ureteral obstruction (UUO). Twenty-four adult male Swiss mice weighing 20-25 g were divided into 4 groups: group $\mathrm{C}(\mathrm{N}=6)$ was not subjected to any surgical manipulation; group $\mathrm{SH}(\mathrm{N}=6)$ was subjected to surgical manipulation but without ureter ligation; group UUO $(\mathrm{N}=6)$ was subjected to unilateral ureteral obstruction and received no treatment; group UUO plus DS $(\mathrm{N}=6)$ was subjected to UUO and received DS $(4 \mathrm{mg} / \mathrm{kg})$ subcutaneously, daily, for 14 days. An immunoblot study was also performed for TGF- $\beta$. Collagen (stained area $\sim 3700 \mu \mathrm{m}^{2}$ ), MCP-1 (stained area $\sim 1700 \mu \mathrm{m}^{2}$ ), TGF- $\beta$ (stained area $\sim 13 \%$ of total area), macrophage (number of cells $\sim 40$ ), and myofibroblast (stained area $\sim 1900 \mu \mathrm{m}^{2}$ ) levels were significantly $(P<0.05)$ higher in the UUO group compared to control. DS treatment significantly $(P<0.05)$ reduced the content of collagen (stained area $\sim 700 \mu \mathrm{m}^{2}$ ), MCP-1 (stained area $\sim 160 \mu \mathrm{m}^{2}$ ) and TGF- $\beta$ (stained area $\sim 5 \%$ of total area), in addition to myofibroblast (stained area $\sim 190 \mu \mathrm{m}^{2}$ ) and macrophage (number of cells $\sim 32$ ) accumulation in the obstructed kidney. Overall, these results indicate that DS attenuates kidney inflammation by reducing macrophage recruitment, myofibroblast population and fibrosis in mice submitted to UUO.
\end{abstract}

Key words: Glycosaminoglycans; Dermatan sulfate; Kidney; Inflammation

\section{Introduction}

Unilateral ureteral obstruction (UUO) is an experimental model used widely to study the pathogenesis of tubulointerstitial fibrosis because it is highly reproducible and induces pathogenic events similar to those observed in human renal fibrosis $(1,2)$.

Renal interstitial fibrosis resulting from ureteral obstruction is associated with the development of an inflammatory process, consisting of chemokine-mediated macrophage recruitment, transforming growth factor $\beta$ (TGF- $\beta$ ) production and myofibroblast accumulation. These events lead to collagen deposition and fibrosis $(3,4)$ and so far no specific treatment is available that can efficiently block or even reverse the progression of renal disease.

Cellular adhesion molecules such as selectins play an essential role in most inflammatory reactions, mediating

Correspondence: M.S.G. Pavão, Laboratório de Bioquímica e Biologia Celular de Glicoconjugados, Hospital Universitário Clementino Fraga Filho, Rua Rodolpho Paulo Rocco, 255, 4º andar, Sala 4A-08, 21941-913 Rio de Janeiro, RJ, Brasil. E-mail: mpavao@hucff.ufrj.br

*These authors contributed equally to this study.

Received February 9, 2011. Accepted June 6, 2011. Available online June 17, 2011. Published July 25, 2011. 
the initial leukocyte rolling events on activated endothelium. During inflammation, P-selectin-glycoprotein-ligand 1 (PSGL-1) on the surface of leukocytes binds to Pselectin on the activated endothelial cells, reducing the velocity of the leukocytes and allowing their contact with endothelial adhesion molecules such as intercellular adhesion molecule 1 (I-CAM-1), and transmigration $(5,6)$. Therefore, it is possible that selectins on the surface of peritubular capillary endothelial cells facilitate leukocyte recruitment to the inflamed renal interstitium.

Heparin is an anticoagulant glycosaminoglycan that acts by activating antithrombin in order to inhibit thrombin and factor $\mathrm{Xa}(7,8)$. It has been recently shown that, in addition to having an anticoagulant effect, heparin inhibits inflammation. The molecular mechanism of this effect involves $\mathrm{P}$ - and L-selectin blockage, which reduces leukocyte recruitment $(9,10)$. Unfractionated heparin has a higher binding affinity for $\mathrm{P}$-selectin when compared to low molecular weight heparin (LMW-Hep) (11). This difference may be associated with the higher effect of unfractionated heparin in suppressing P-selectin function in vivo (12). In fact, we recently reported that LMW-Hep attenuates fibrosis in obstructed kidneys by down-regulating the synthesis of collagen, fibronectin and TGF- $\beta$, but it is not able to inhibit macrophage recruitment (13).

Dermatan sulfate (DS) is a sulfated glycosaminoglycan consisting of alternating units of $\mathrm{N}$-acetyl-Dgalactosamine (GalNAc) and iduronic acid (IdoA), which can be sulfated mainly at the carbon 4 (C4) of GalNAc and the carbon 2 (C2) of the IdoA residues (14). DS has been successfully used in humans to treat and prevent heparin-induced thrombocytopenia (15). It has also been shown to bind and block P- and L-selectin function in vitro $(16,17)$. Recently, we reported that subcutaneous administration of DS attenuates colon inflammation in rats by reducing macrophage and $T$-cell recruitment and macrophage activation (18), suggesting its potential use as an anti-inflammatory drug.

In the present investigation, we extended the study of the anti-inflammatory effect of DS by examining its effect in mice after UUO by measuring the content of monocyte chemoattractant protein-1 (MCP-1) and TGF- $\beta$, as well as macrophage, myofibroblast and collagen accumulation in the kidney. The results indicate that DS has a protective effect against the progression of kidney inflammation by reducing macrophage and myofibroblast accumulation and fibrosis.

\section{Material and Methods}

\section{Animals and experimental protocol}

All animal work was carried out in accordance with the Brazilian Animal Protection Law and the Ethics Committee for Animal Use of Universidade Federal do Rio de Janeiro. The study was performed on 24 adult male Swiss mice weighing 20-25 g. The mice were kept on a $12-\mathrm{h}$ light/dark cycle at $25^{\circ} \mathrm{C}$ and fed a standard mice chow and water ad libitum. Ureteral obstruction was performed as follows: mice were anesthetized subcutaneously with ketamine (35 mg/kg) and xylazine (5 $\mathrm{mg} / \mathrm{kg}$ ). Under sterile conditions, an abdominal midline incision was made, the left ureter was exposed and ligated at two points using 6-0 silk, and the ureter was sectioned between the ligatures. The skin was sutured for approximation and the mice were kept in regular cages. The mice were divided into 4 groups. A group of 6 mice served as control and was not subjected to any surgical manipulation (group $\mathrm{C}$ ). The $\mathrm{SH}$ group (shamoperated animals, $\mathrm{N}=6$ ) was subjected to the surgical procedures, except that the left ureter was manipulated without ligation and sectioning. The UUO group ( $N=$ 6 ) was subjected to ureteral obstruction and given no treatment. Another group of 6 mice was subjected to ureteral obstruction and was given porcine intestinal mucosa DS (4 mg $\mathrm{kg}^{-1} \cdot \mathrm{day}^{-1}$, dissolved in $0.30 \mathrm{~mL}$ saline; Sigma, USA) subcutaneously, once daily (group UUO+DS) for 14 days.

\section{Tissue preparation}

At the end of 14 days, the mice were sacrificed under anesthesia and the kidneys were removed. Animals were perfused with citrate buffer $(3.5 \%)$, pH 7.4, via the left cardiac ventricle for $20 \mathrm{~min}$. Kidneys were removed and sectioned mid-frontally into two pieces. One fragment was immersed in $10 \%$ buffered formaldehyde solution and embedded in paraffin for histological examination and the other was immersed in acetone for hydroxyproline analysis. Kidney paraffin sections of $5 \mu \mathrm{m}$ were stained with hematoxylin-eosin ( $\mathrm{HE})$, and a modified Sirius red technique $(19,20)$ for collagen staining. Tissue samples were immediately embedded in Tissue-Tek O.C.T. compound (Miles Scientific Laboratories Ltd., USA) and snap-frozen in isopentane in a liquid nitrogen bath. Samples were then stored at $-80^{\circ} \mathrm{C}$ until processing, and cut into $6-\mu \mathrm{m}$ sections with a cryostat maintained at $-20^{\circ} \mathrm{C}$. Tissue sections were air-dried and fixed for $10 \mathrm{~min}$ in 1:1 chloroform-acetone. For the detection of macrophages and TGF- $\beta$, a rat anti-mouse F4/80 antibody (1:1000; Serotec, USA) and a pan-specific rabbit anti-TGF- $\beta$ antibody (1:100; R\&D Systems, USA) were used, respectively (18). Antibodies were detected with the Rat IgG kit (Vector Labs, USA) for F4/80, and the Histar 40000 mouse on Mouse kit (Serotec) for TGF- $\beta$ and developed with the Dako LSAB ${ }^{\circledR} 2$ kit/horseradish peroxidase (HRP; Dako Corp., USA) using diaminobenzidine (DAB) as the chromogen substrate (Liquid DAB; Dako).

Interstitial myofibroblasts were quantified using an $\alpha$-smooth muscle actin ( $\alpha-S M A)$ monoclonal antibody (1:100; Dako Corp.). An anti-MCP-1 polyclonal antibody 
(1:100; Santa Cruz Biotechnology) was used for MCP-1 detection. For these immunohistochemical stainings, an avidin-biotin HPS system (Vector Laboratories) was used with $\mathrm{DAB}$ to yield a brown-colored end-product at the site of detection. Sections were then counterstained with $\mathrm{HE}$, dehydrated, and mounted.

\section{Histomorphometry}

Histomorphometry was performed using an imaging analysis system consisting of a digital camera (Coolpix 990, Nikon, Japan) coupled to a light microscope (Eclipse 400 , Nikon). Fifteen fields of renal cortex and of medulla from Sirius red-stained sections, as well as sections stained with anti-F4/80 and anti-TGF- $\beta$, anti-MCP-1 and anti- $\alpha$-SMA antibodies were obtained for each animal, using a 40X magnification objective lens.

The immunostaining was quantified as stained area or number of cells $\left(\mu \mathrm{m}^{2}\right)$ or as cells associated with a brownish color around the nucleus. There is a clear distinction between nuclei associated with immunostaining and those devoid of the brownish color. Quantification was estimated on captured high-quality images (2048 x 1536 pixels buffer) by considering the percentage of stained areas in the total histological fields using the Image Pro Plus 4.5.1 software (Media Cybernetics, USA).

\section{Determination of hydroxyproline}

The amount of hydroxyproline (the collagen-specific hydroxylated form of the amino acid proline) in the renal tissue was estimated by a modification of the method of Stegemann and Stalder (21). Briefly, the samples were immersed in acetone for $24 \mathrm{~h}$ at $4^{\circ} \mathrm{C}$ and dried in an oven at $60^{\circ} \mathrm{C}$. About $30 \mathrm{mg}$ of the dried material was subjected to acid hydrolysis with $6 \mathrm{~N}$ hydrochloric acid $(\mathrm{HCl})$ at $107^{\circ} \mathrm{C}$ for $18 \mathrm{~h}$. $\mathrm{HCl}$ was then removed by evaporation and the hydrolyzed material was mixed with $200 \mu \mathrm{L}$ buffer $\left(5 \%\right.$ citric acid. $1 \mathrm{H}_{2} \mathrm{O}, 1.2 \%$ acetic acid, $12 \%$ sodium acetate. $3 \mathrm{H}_{2} \mathrm{O}$, and $3.4 \%$ sodium hydroxide, $\mathrm{pH}$ 6.0) diluted 1:10. The mixture was incubated with $1 \mathrm{~mL}$ chloramine-T solution for $20 \mathrm{~min}$ at room temperature and $1 \mathrm{~mL}$ aldehyde/perchloric acid solution was added and incubated for another $15 \mathrm{~min}$ at $60^{\circ} \mathrm{C}$. Absorbance at $570 \mathrm{~nm}$ was recorded after $20 \mathrm{~min}$. The concentration was estimated with a standard curve using a solution of analytical grade hydroxyproline.

\section{Immunoblotting for TGF- $\beta$}

Kidney slices were quickly washed in ice-cold phosphate-buffered saline (PBS), minced, and $50 \mathrm{~mL}$ buffer (10\% sodium dodecyl sulfate (SDS), $20 \%$ glycerol, $0.2 \mathrm{M}$ dithiothreitol, $20 \mathrm{mM}$ Tris- $\mathrm{HCl}, \mathrm{pH} 6.8$ ) was added to the extracts. Kidney extracts were recovered in a tube, centrifuged, and boiled for $10 \mathrm{~min}$. Samples were submitted to $10 \%$ SDS-polyacrylamide gel electrophoresis (SDS-PAGE). Proteins were transferred to polyvinylidenefluoride membranes (Millipore, Brazil). The proteins immobilized on the membranes were immediately blocked for $1 \mathrm{~h}$ at room temperature with a solution of $5 \%$ nonfat milk in Tris-buffered saline-Tween $20(0.001 \%$ TBS-T). The membranes were then incubated with a pan-specific rabbit anti-TGF- $\beta$ antibody $(1.5 \mu \mathrm{g} / \mathrm{mL}$; R\&D Systems). After 5 washes in TBS-T (3 min each), the membranes were incubated with an anti-rabbit peroxidase-conjugated antibody (1:1000 in TBS-T; Promega, USA), washed again as described above, and the bands were developed using the West Pico Pierce kit (Pierce, USA).

To check sample loading, membranes were treated with striping buffer ( $2 \%$ SDS, $100 \mathrm{mM}$ 2-mercaptoethanol, $62.5 \mathrm{mM}$ Tris- $\mathrm{HCl}, \mathrm{pH} 6.7$ ) for $30 \mathrm{~min}$ at $50^{\circ} \mathrm{C}$, washed five times in TBS-T, and blocked again as described above. The membranes were then incubated with a rabbit polyclonal anti-actin antibody (1:3000 in TBS-T; Sigma). After 5 washes in TBS-T ( 3 min each), the membranes were incubated with an anti-rabbit peroxidase-conjugated antibody (1:1000 in TBS-T-milk) and developed as described above.

\section{Statistical analysis}

Data are reported as means \pm SD or median and range. Comparisons between groups were done by one-way ANOVA, or by the Kruskal-Wallis test followed by the Tukey post-test, with the level of significance set at $P<0.05$.

\section{Results}

\section{Morphological changes in obstructed kidneys}

The effect of subcutaneous administration of DS on the progression of renal disease was examined in mice. Histological analysis of HE-stained sections revealed an evident increase in cellular infiltration in obstructed kidneys (Figure 1). DS administration to obstructed mice clearly attenuated cellular infiltration.

\section{Collagen}

Collagen content was estimated by histomorphometry of Sirius red-stained sections and by measuring hydroxyproline in renal tissues (Figure 2), as described in Material and Methods. The histomorphometry of Sirius red-stained sections, reported as $\mu \mathrm{m}^{2}$ (Figure $2 \mathrm{~A}$ and $B)$, revealed a $\sim 7.7$-fold increase in collagen deposition in the interstitium of obstructed kidneys (group UUO; median value $=3717.1$ ) compared to group $C$ (median 494.90; $P<0.05$ ). No significant difference was observed between groups $\mathrm{C}, \mathrm{SH}$, and UUO+DS (median value = 1153.5). Collagen deposition was lower in the UUO+DS group than in the UUO group (median value $=3717.1$; $P<0.05$ ), representing a $\sim 3.2$-fold reduction.

Determination of the hydroxyproline content $(\mu \mathrm{g} /$ 
mg tissue dry weight; Figure $2 \mathrm{C}$ ) revealed a $\sim 3.2$-fold increase in obstructed kidneys (group UUO; median value $=3.21$ ) compared to non-obstructed kidneys (group C) $($ median value $=1.0 ; P<0.05)$. Daily subcutaneous administration of DS for 14 days to mice after UUO significantly reduced hydroxyproline content in the

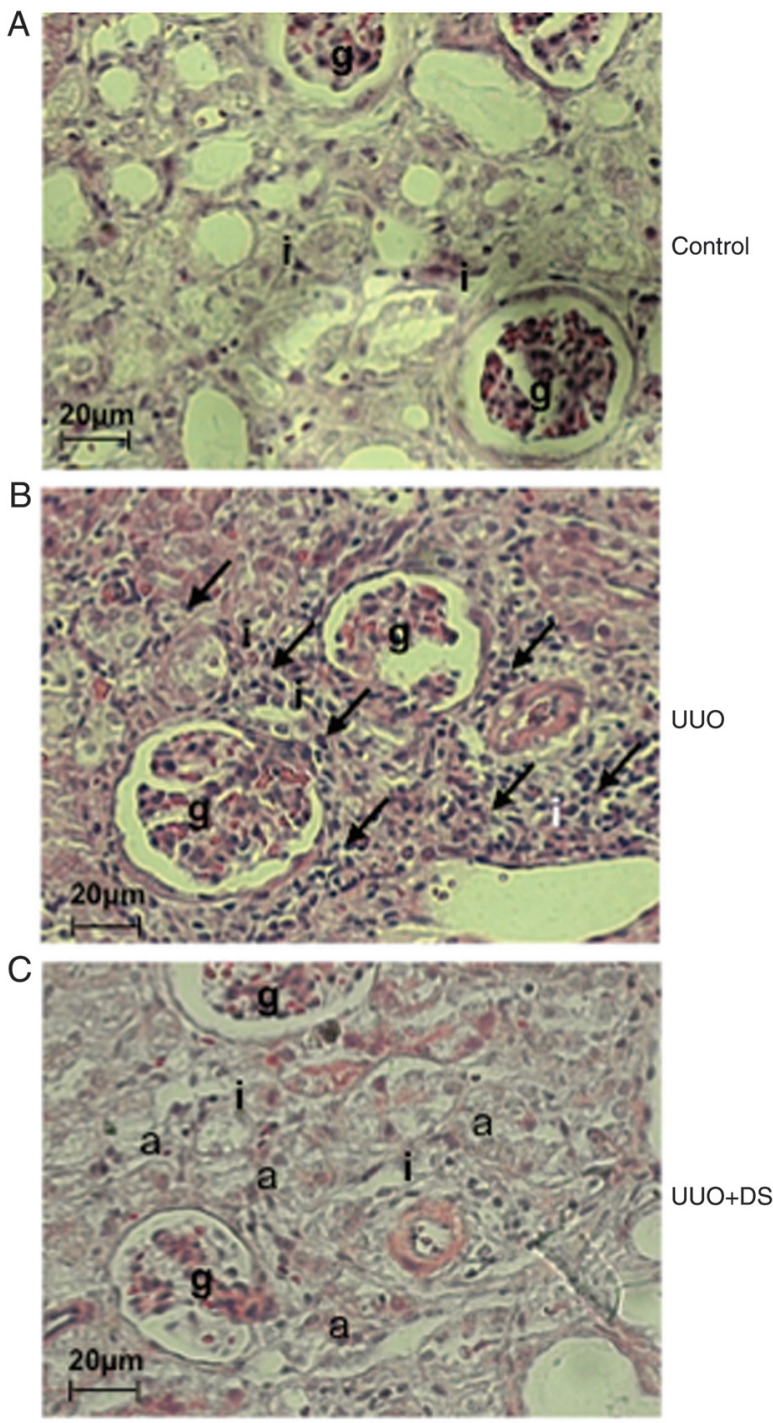

Figure 1. Histochemical analysis of hematoxylin-eosin (HE)stained sections from obstructed kidneys of mice submitted to dermatan sulfate (DS) treatment. $A$, HE-stained section from the control group. $B$, HE-stained section from the group with unilateral ureteral obstruction (UUO, obstructed kidney with no treatment). C, HE-stained section of obstructed mouse kidneys from the group with unilateral ureteral obstruction plus dermatan sulfate (UUO+DS, obstructed kidney treated with $4 \mathrm{mg} \cdot \mathrm{kg}^{-1} \cdot \mathrm{day}^{-1}$ DS for 14 days). Arrows indicate inflammatory cells in the interstitium. Note the reduced inflammatory cells in the interstitium of obstructed kidney from animals of group UUO+DS (a). g = glomerulum; i = interstitium. Scale bar $=20 \mu \mathrm{m}$. Magnification 400X. interstitial space of obstructed kidneys (group UUO+DS) to a median value of 1.7 compared to group UUO $(\mathrm{P}<$ 0.05 ; Figure 2C).

\section{Myofibroblast accumulation}

The increased synthesis of extracellular matrix at the site of inflammation is associated with the accumulation of myofibroblast in the renal interstitium. Thus, we questioned whether the effect of DS on collagen deposition was related to a decrease in myofibroblast accumulation in the kidney. The myofibroblast content was estimated by histomorphometry of $\alpha$-SMA-stained renal sections (Figure $3 \mathrm{~A}$ and $\mathrm{B}$ ). When compared to group $\mathrm{C}$, renal sections of obstructed mice (group UUO) showed a $\sim 10$ fold increase in myofibroblast accumulation $(P<0.05)$. Administration of DS to obstructed mice significantly reduced myofibroblast accumulation (group UUO+DS) to group $C$ values $(P<0.05)$. No significant difference was observed between groups $\mathrm{C}$ and UUO+DS.

\section{Macrophage infiltration}

To investigate the effect of DS on macrophage infiltration, sections from control and obstructed kidneys were stained with the rat anti-mouse monoclonal antibody F4/80. Thirteen fields of renal cortex and medulla from F4/80-stained sections were captured from each animal and the results are reported as the number of macrophages per field. F4/80-positive cells increased from a median value of 0.816 for group $C$ to 44.26 for group UUO ( $P<0.05$; Figure 4A and $B$ ). Administration of DS to mice after UUO decreased macrophage infiltration (median value $=19.8 ; \mathrm{P}<0.05$ ). There was no significant difference between groups $\mathrm{C}$ and $\mathrm{SH}$.

\section{Monocyte chemoattractant protein-1}

The recruitment of macrophages to the inflamed kidney depends on the release of several chemokines, including MCP-1. Therefore, we investigated the production of MCP-1 in obstructed kidneys after DS administration. The content of MCP-1 was estimated by immunohistomorphometry of renal tissue using an anti-MCP-1 antibody (Figure 5A and $\mathrm{B}$ ). When compared to group $\mathrm{C}$, renal sections of obstructed mice (group UUO) showed a drastic increase in MCP-1 content. Administration of DS to obstructed mice significantly reduced MCP-1 content (group UUO+DS) to control values $(P<0.05)$. No significant difference was observed between groups $\mathrm{C}$ and UUO+DS.

\section{Transforming growth factor- $\beta$}

TGF- $\beta$ plays a crucial role in the progression of renal fibrosis associated with the model of UUO. The histomorphometry of stained sections, expressed as percent surface area, revealed, as expected, a $\sim 6.5$-fold increase, showing a uniform distribution within cortical 
A
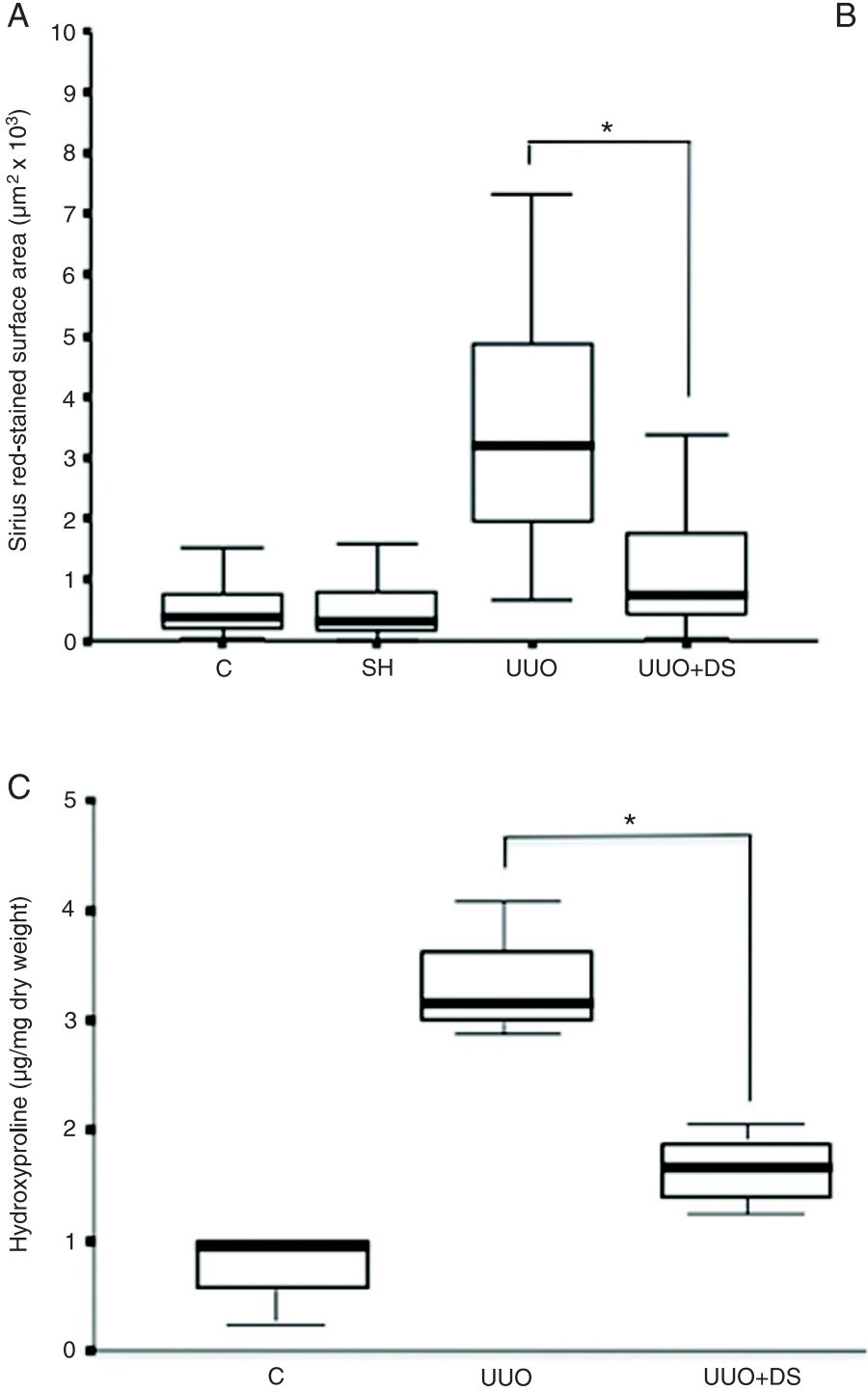

B

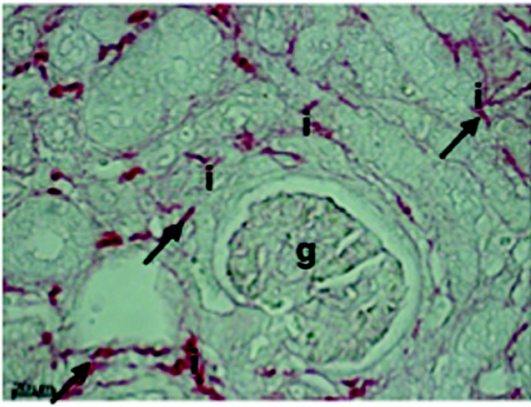

Control

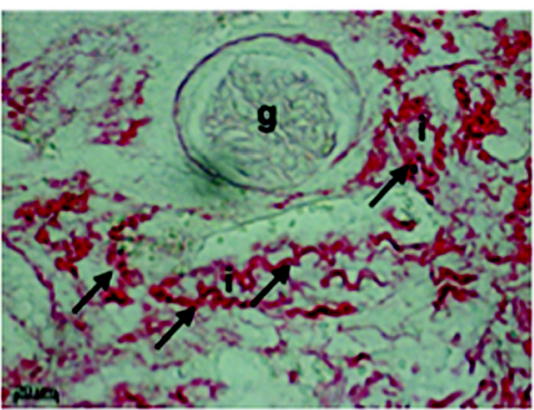

UUO

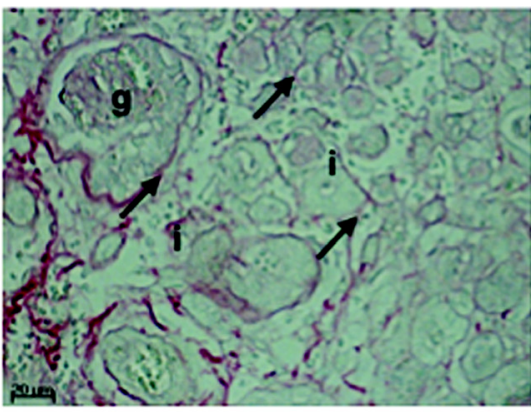

UUO+DS

Figure 2. Collagen content in kidneys from mice submitted to different treatments. $A$, Histomorphometry of Sirius red-stained sections of kidneys of mice from groups $\mathrm{C}$ (control kidney), $\mathrm{SH}$ (sham-operated kidney), unilateral ureteral obstruction (UUO, obstructed kidney with no treatment), and unilateral ureteral obstruction plus dermatan sulfate (UUO+DS, obstructed kidney treated with $4 \mathrm{mg} \cdot \mathrm{kg}^{-1} \cdot \mathrm{day}^{-1}$ DS for 14 days). The horizontal bars represent the medians, boxes represent the 25th and 75th percentiles, and the vertical bars represent the ranges, $N=30$. $B$, The arrows in the UUO panel point to dense Sirius red-stained areas compared with those with reduced stained areas in control and UUO+DS panels. $C$, Hydroxyproline $(\mu \mathrm{g} / \mathrm{mg}$ dry weight) was used to estimate collagen content of mouse kidneys from group C, group SH, group UUO, and group UUO+DS. The horizontal bars represent the medians, the boxes represent the 25th and 75th percentiles, and the vertical bars represent the ranges, $\mathrm{N}=30 . \mathrm{g}=$ glomerulum; $\mathrm{i}=$ interstitium. Magnification 400X. Scale bar $=20 \mu \mathrm{m} .{ }^{*} \mathrm{P}<0.05$ (ANOVA).

and medullary tubules in the obstructed kidneys of mice after UUO (group UUO; median value $=14.11$ ) compared to group $C$ (median value $=2.51 ; \mathrm{P}<0.05:$ Figure $4 \mathrm{~A}$ and B). Group UUO+DS (median value $=6.38$ ) showed a much lower content of TGF- $\beta$ compared to group UUO $(P<0.05)$ (Figure $6 A$ and $B)$. There was no significant difference between groups $\mathrm{SH}, \mathrm{C}$, and C+DS. Western blot analysis confirmed our immunohistochemical results for TGF- $\beta$. There was an increase in the amount of TGF- $\beta$ in obstructed kidneys compared to control. DS treatment drastically reduced TGF- $\beta$ in obstructed kidneys (Figure 6C). 

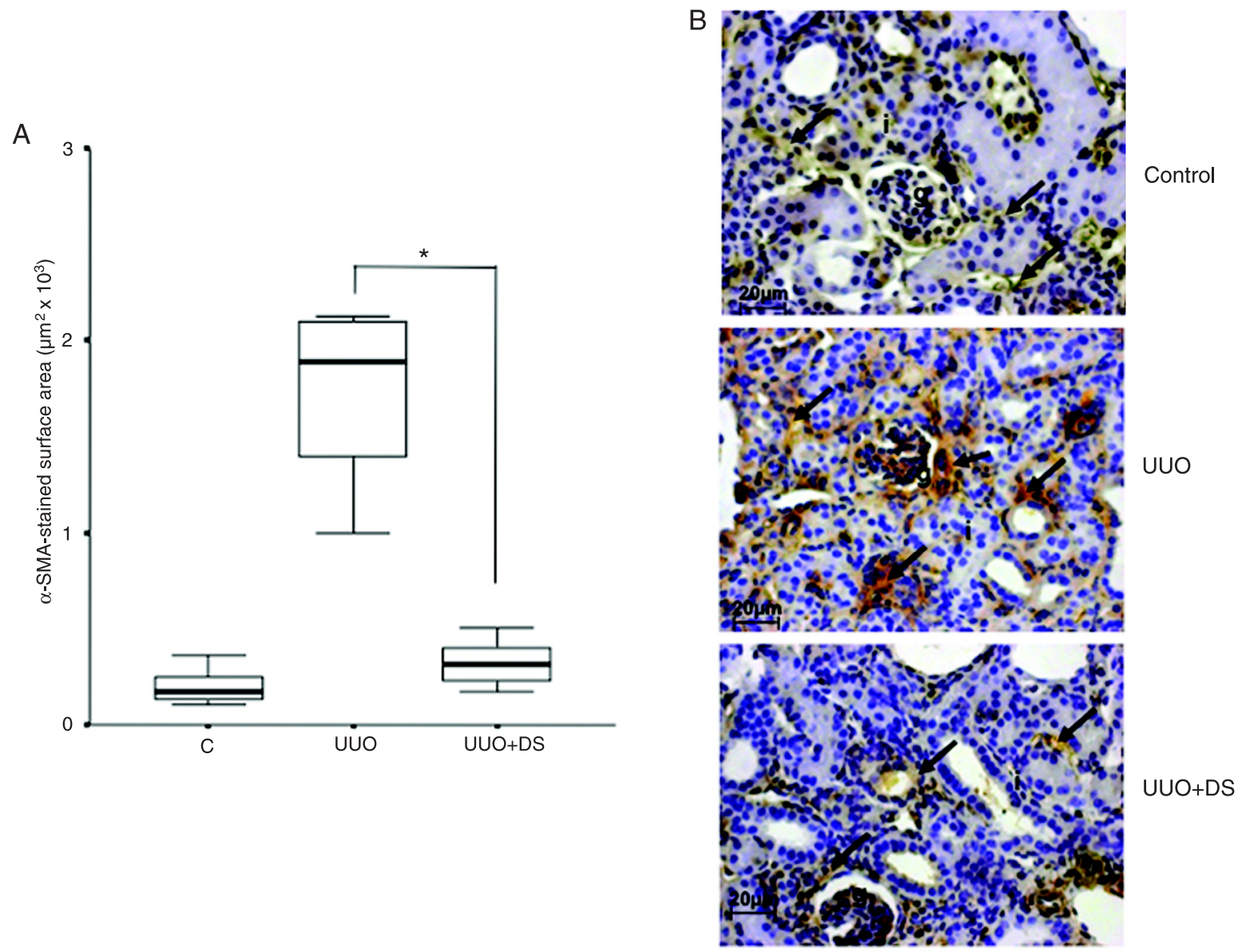

Figure 3. Myofibroblast accumulation in the kidney of mice submitted to different treatments. $A$, Myofibroblast accumulation was esti-

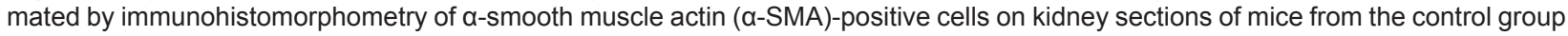
(C), unilateral ureteral obstruction (UUO) group and unilateral ureteral obstruction plus dermatan sulfate group (UUO+DS). Data are reported as means $\pm S D$ for $N=6$. The boxes represent the 25th and 75 th percentiles, and the vertical bars represent the ranges, $N=$ 30. $B$, The arrows in the UUO panel depict areas strongly stained for a-SMA compared with those less stained in control and UUO+DS panels. $g=$ glomerulum; $i=$ interstitium. Scale bar $=20 \mu \mathrm{m}$. Magnification $40 \mathrm{X} .{ }^{*} \mathrm{P}<0.05$ (ANOVA).

\section{Discussion}

Kidney diseases can often lead to end-stage renal failure, which has a high morbidity and mortality. The prevalence of this condition is increasing at a rate of $\sim 7 \%$ annually worldwide. Although several mechanisms involved in the process of fibrogenesis have been identified, no specific treatment is available that can efficiently block or reverse the progression of renal disease.

The UUO model of fibrosis has been well established and is currently used in an extensive manner (3). It is characterized by renal myofibroblast activation, tubular atrophy, and interstitial fibrosis. Myofibroblast accumulation is characterized by an increase in the content of extravascular $\alpha-S M A$. This can be the result of either resident fibroblast transdifferentiation, bone marrow- derived cells or epithelial to mesenchymal transition (4). After UUO, TGF- $\beta$ and its receptor are up-regulated in the renal tubular epithelium. Increased cellular infiltration, tubular atrophy and extracellular deposition are also present (3).

In the present study, we investigated the effect of DS on renal inflammation in mice submitted to UUO. The mice were treated by daily subcutaneous injection of DS (4 mg/kg) for 14 days. Ureteral obstruction produced the expected inflammatory response including morphological remodeling of the kidney, evidenced by tubular damage, interstitial edema and increased cellular recruitment. Increased TGF- $\beta$ and MCP-1 production, collagen deposition, as well as myofibroblast accumulation, were also observed. 

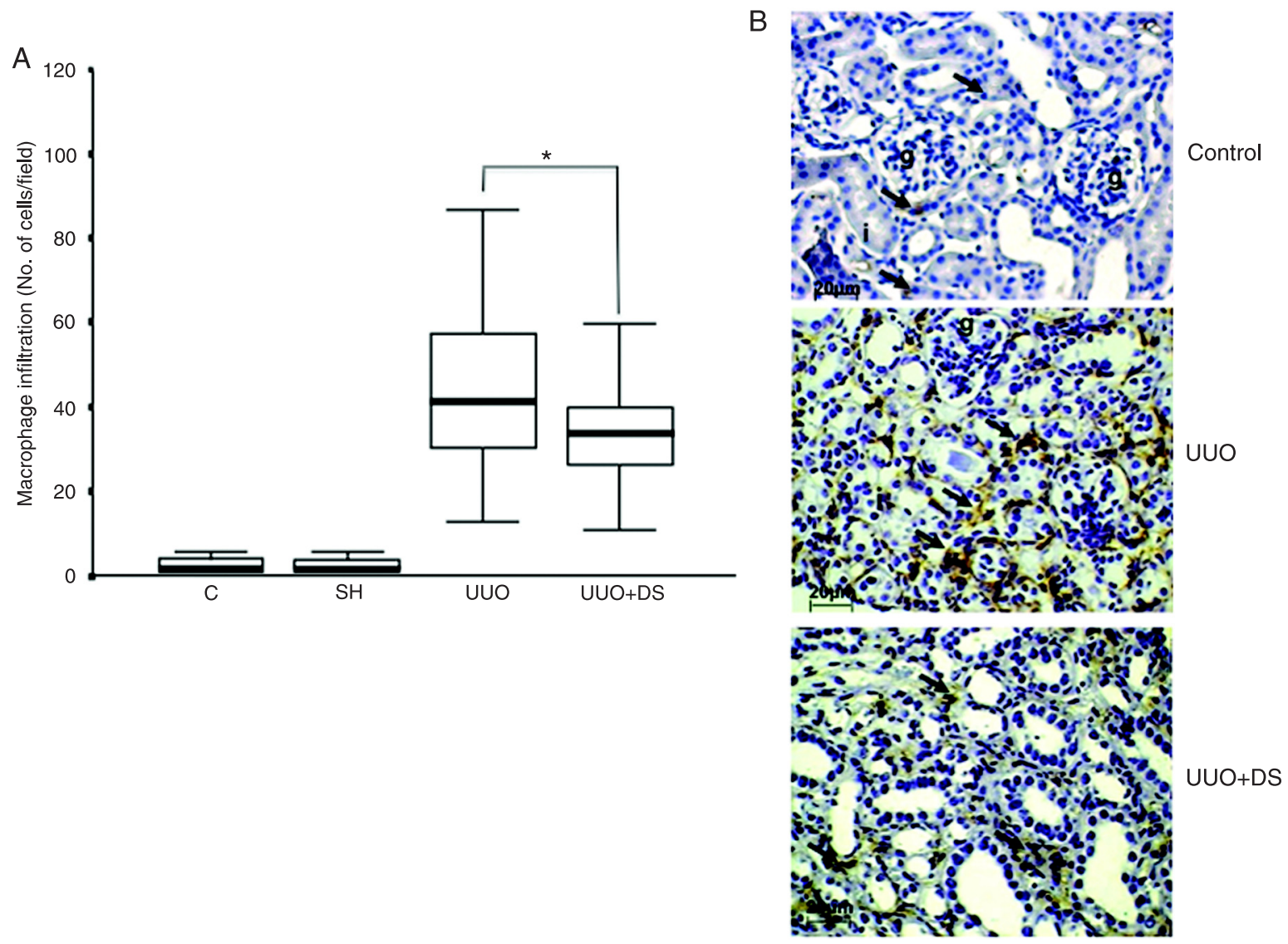

Figure 4. Macrophage infiltration into the kidney of mice submitted to different treatments. $A$, Macrophage infiltration was estimated by immunohistomorphometry of F4/80-positive cells in sections of kidneys of mice from group C (control kidney), group SH (sham-operated kidney), group unilateral ureteral obstruction (UUO), and group unilateral ureteral obstruction plus dermatan sulfate (UUO+DS). Data are reported as means $\pm S D$ for $N=6$. The boxes represent the 25th and 75th percentiles, and the vertical bars represent the ranges, $\mathrm{N}=30$. $B$, The arrows point to strong $\mathrm{F} 4 / 80$-positive cells in the interstitial area compared with those with no staining in control and UUO+DS panels. $g=$ glomerulum; $\mathrm{i}=$ interstitium. Scale bar $=20 \mu \mathrm{m}$. Magnification 400X. * $\mathrm{P}<0.05$ (ANOVA).

DS administration reduced tubular damage and fibrosis, as indicated by histological analysis of the obstructed kidney. This protection may be the result of combined effects of DS treatment, such as reduced TGF- $\beta$ production, since this cytokine is involved in the synthesis of matrix components, including collagen, during the inflammatory process (22); reduced epithelial to mesenchymal transition, as indicated by the reduction in myofibroblast accumulation; reduced production of MCP-1 and macrophage recruitment.

The recruitment of mononuclear cells from blood to inflamed tissues requires several chemokines, such as MCP-1, macrophage inflammatory protein 1 , and RANTES (3), and involves the interaction of endothelial P-selectin on the surface of activated endothelium, and P-selectin glycoprotein ligand-1 (PSGL-1), which is the common ligand of P-selectin on leukocytes. DS have been shown to bind P-selectin (16) and to inhibit $P$-selectin function in vitro (17). Therefore, in the context of the present study, exogenous DS that circulates in the peritubular capillary endothelium may act by inhibiting P-selectin-dependent mononuclear cell recruitment to the inflamed kidney. However, additional experiments are needed to confirm this hypothesis.

Inflammatory cells elaborate several molecules such as tumor necrosis factor- $\alpha$ (TNF- $\alpha$ ) and interleukin-1 (IL1) during the fibrogenic-signaling phase of progressive renal disease (3). Binding of these signaling molecules to their cognate receptors expressed by resident kidney cells and infiltrating leukocytes induces the synthesis of TGF- $\beta$, which results in fibrogenesis. DS has been shown to bind several chemokines, including IL- 1 and TGF- $\beta$, and 

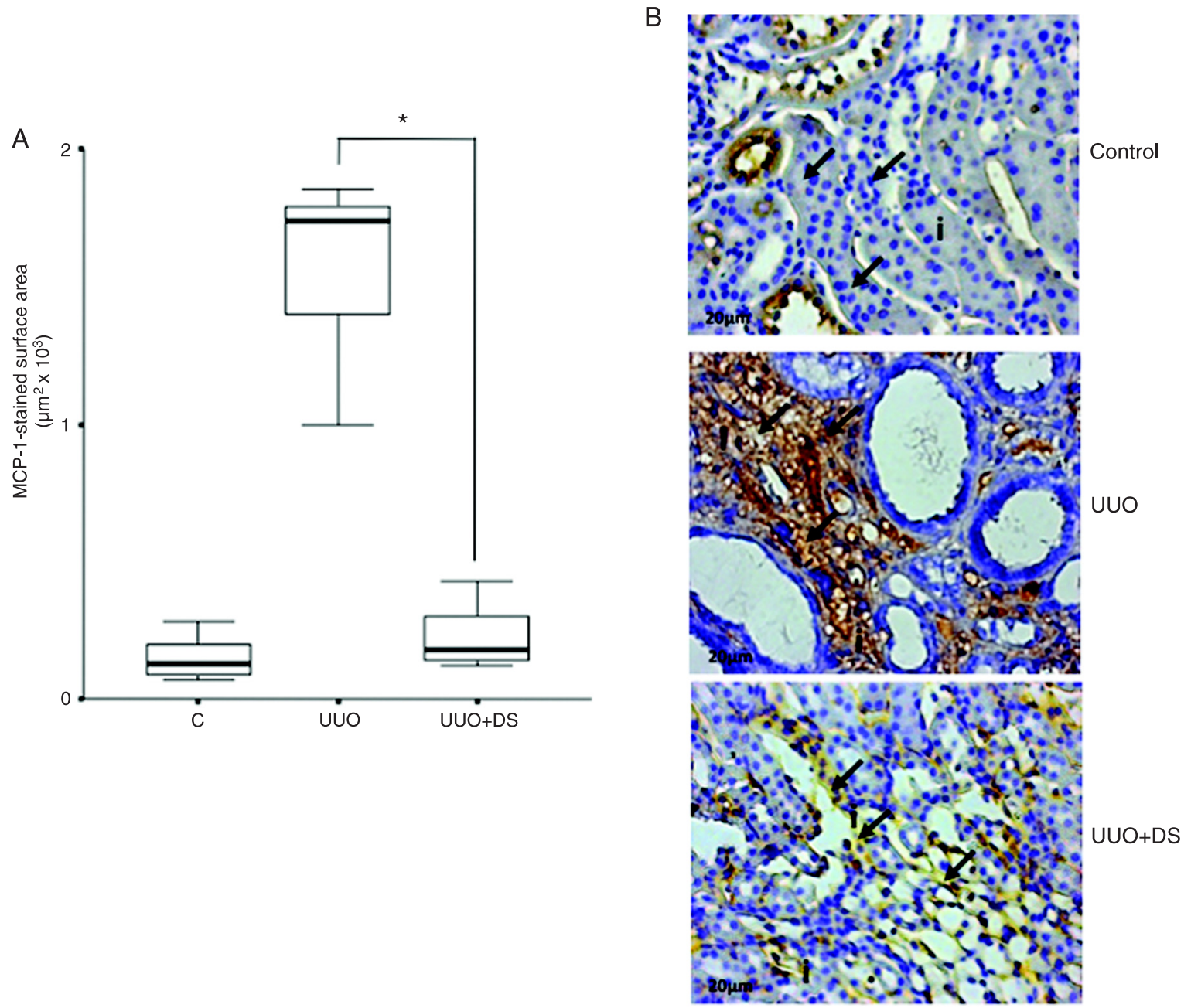

Figure 5. Monocyte chemoattractant protein-1 (MCP-1) content in kidneys from mice submitted to different treatments. $A$, Immunohistomorphometry of anti-MCP-1-stained sections of kidneys of mice from group C (control kidney), group unilateral ureteral obstruction (UUO), and group unilateral ureteral obstruction plus dermatan sulfate (UUO+DS). The horizontal bars represent medians, the boxes represent the 25th and 75th percentiles, and the vertical bars represent the ranges, $N=30$. $B$, The arrows in the UUO panel point to strong MCP-1-stained interstitial areas compared with those with less intense staining in control and UUO+DS panels. $i=$ interstitium. Scale bar $=20 \mu \mathrm{m}$. Magnification 400X. ${ }^{*} \mathrm{P}<0.05$ (ANOVA).

to affect their cellular responses $(23,24)$. Therefore, it is possible that exogenous DS from the plasma that eventually enter the renal interstitial space by the anion transport system (25) could attenuate the various chemokinesignaling events involved in the fibrinogenic process. For example, administration of DS could affect the binding of IL-1, TNF- $\alpha$ or MCP-1 to their receptors on the target cells, inhibiting matrix accumulation along the tubular basement membrane and within the interstitial space.

Overall, the results of the present study indicate that DS attenuates kidney inflammation and fibrosis by reducing macrophage recruitment, myofibroblast accumulation and TGF- $\beta$ expression. Therefore, considering the lower blood clotting effects of DS compared to heparin, and its significant anti-inflammatory effect, this glycan is a potential therapeutic option for the treatment of renal inflammation and fibrosis.

\section{Acknowledgments}

We thank Alison R. Junior and Cesônia A. Martinusso, Laboratório Multidisciplinar, Hospital Universitário Clementino Fraga Filho, UFRJ, for technical support. Research supported by CNPq, FAPERJ, the NIH Fogarty International Center (\#R03 TW05775, to M.S.G. Pavão), and the Mizutani Foundation for Glycoscience (\#RN:080002, to M.S.G. Pavão). M.S.G. Pavão is the recipient of a CNPq research fellowship. 


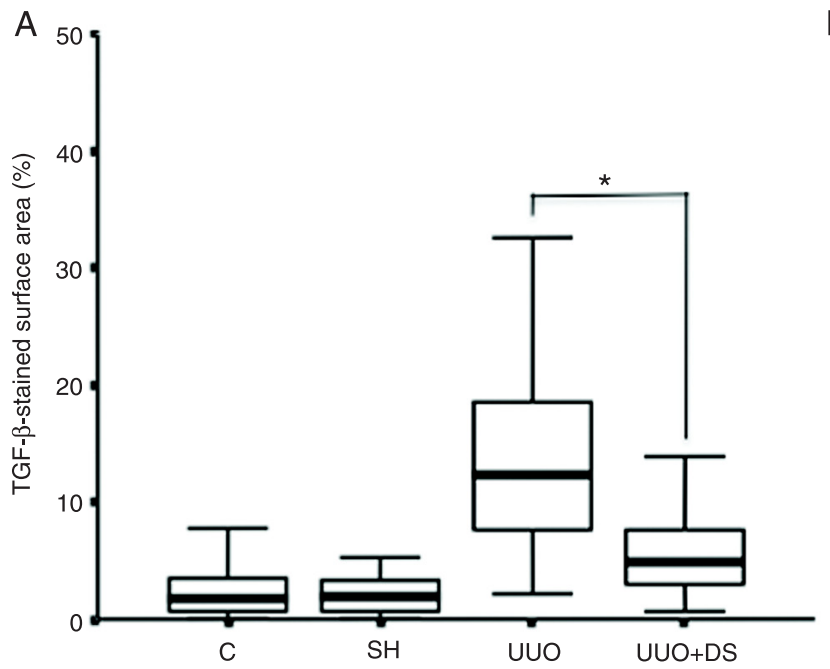

B
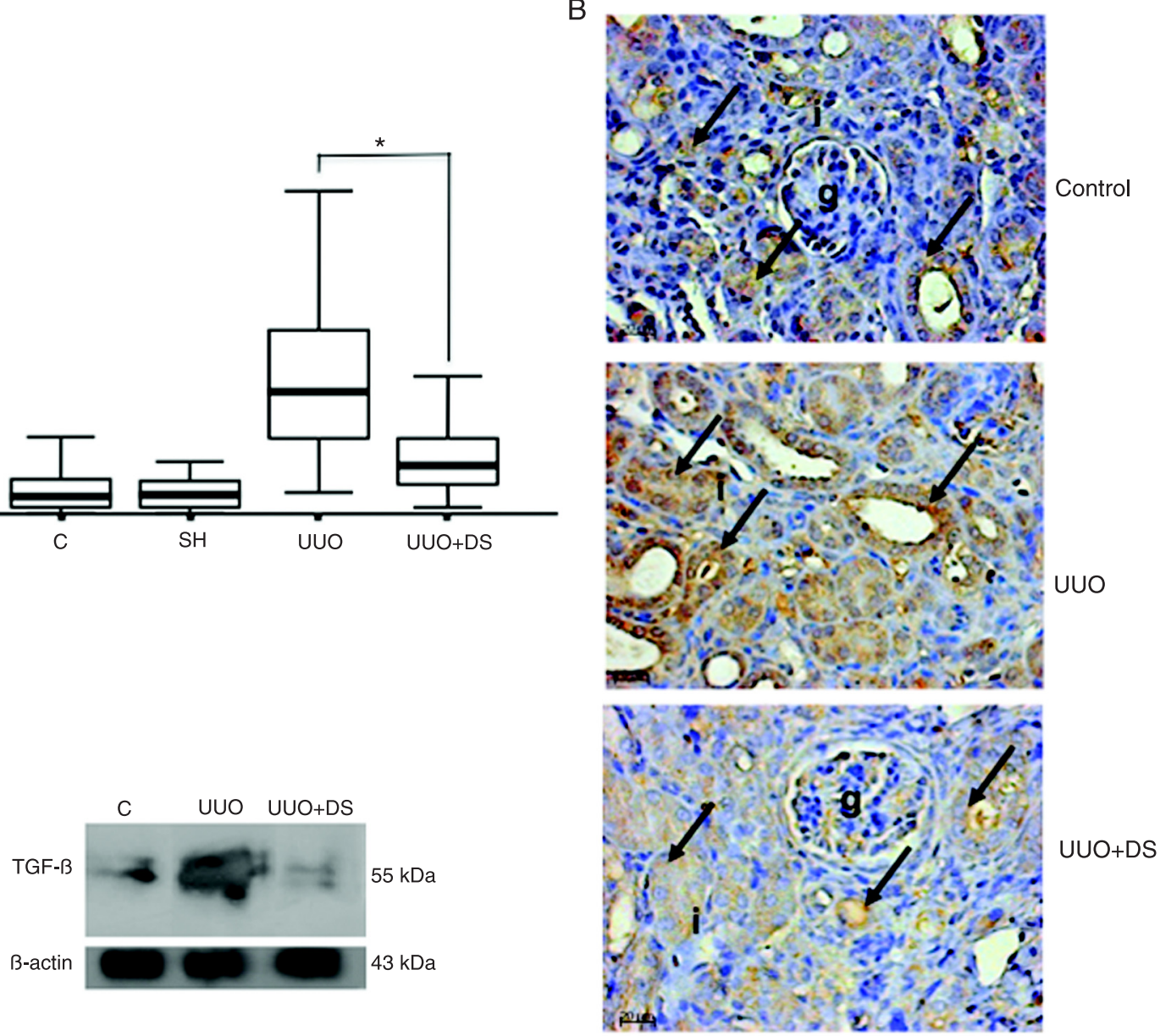

Figure 6. Transforming growth factor beta (TGF- $\beta$ ) content in kidneys from mice submitted to different treatments. $A$, Immunohistomorphometry of anti-TGF- $\beta$-stained sections of kidneys of mice from group $\mathrm{C}$ (control kidney), group sham-operated kidney (SH), group unilateral ureteral obstruction (UUO), and group unilateral ureteral obstruction plus dermatan sulfate (UUO+DS). The horizontal bars represent medians, the boxes represent the 25th and 75th percentiles, and the vertical bars represent the ranges, $N=30$. $B$, The arrows in the UUO panel show strong TGF- $\beta$-stained areas that seem to be near epithelial tubular cells. Note the less intense staining in control and UUO+DS panels. $g$ = glomerulum; $i=$ interstitium. Magnification 400X. $C$, The content of TGF- $\beta$ in rat kidney was analyzed by Western blot using anti-TGF- $\beta$. The amount of the load was normalized by the content of actin, as described in Material and Methods. Scale bar $=20 \mu \mathrm{m} .{ }^{*} \mathrm{P}<0.05$ (ANOVA).

\section{References}

1. Muller GA, Zeisberg M, Strutz F. The importance of tubulointerstitial damage in progressive renal disease. Nephrol Dial Transplant 2000; 15 (Suppl 6): 76-77.

2. Schaefer L, Macakova K, Raslik I, Micegova M, Grone HJ, Schonherr E, et al. Absence of decorin adversely influences tubulointerstitial fibrosis of the obstructed kidney by enhanced apoptosis and increased inflammatory reaction. Am J Pathol 2002; 160: 1181-1191.

3. Eddy AA. Molecular basis of renal fibrosis. Pediatr Nephrol 2000; 15: 290-301.

4. Lange-Sperandio B, Trautmann A, Eickelberg O, Jayachan- dran A, Oberle S, Schmidutz F, et al. Leukocytes induce epithelial to mesenchymal transition after unilateral ureteral obstruction in neonatal mice. Am J Pathol 2007; 171: 861871.

5. McEver RP, Moore KL, Cummings RD. Leukocyte trafficking mediated by selectin-carbohydrate interactions. J Biol Chem 1995; 270: 11025-11028.

6. Lasky LA. Selectin-carbohydrate interactions and the initiation of the inflammatory response. Annu Rev Biochem 1995; 64: 113-139.

7. Rosenberg JS, Rosenberg RD. Advances in the understand- 
ing of the anticoagulant function of heparin. Curr Ther Res Clin Exp 1975; 18: 66-78.

8. Rosenberg RD. Biochemistry and pharmacology of low molecular weight heparin. Semin Hematol 1997; 34: 2-8.

9. Gao Y, Li N, Fei R, Chen Z, Zheng S, Zeng X. P-Selectinmediated acute inflammation can be blocked by chemically modified heparin, RO-heparin. Mol Cells 2005; 19: 350-355.

10. Wang L, Brown JR, Varki A, Esko JD. Heparin's anti-inflammatory effects require glucosamine 6-O-sulfation and are mediated by blockade of L- and P-selectins. J Clin Invest 2002; 110: 127-136.

11. Simonis D, Christ K, Alban S, Bendas G. Affinity and kinetics of different heparins binding to P- and L-selectin. Semin Thromb Hemost 2007; 33: 534-539.

12. Ludwig RJ, Alban S, Bistrian R, Boehncke WH, Kaufmann R, Henschler R, et al. The ability of different forms of heparins to suppress $\mathrm{P}$-selectin function in vitro correlates to their inhibitory capacity on bloodborne metastasis in vivo. Thromb Haemost 2006; 95: 535-540.

13. Pecly IM, Goncalves RG, Rangel EP, Takiya CM, Taboada FS, Martinusso CA, et al. Effects of low molecular weight heparin in obstructed kidneys: decrease of collagen, fibronectin and TGF-beta, and increase of chondroitin/dermatan sulfate proteoglycans and macrophage infiltration. Nephrol Dial Transplant 2006; 21: 1212-1222.

14. Pavao MS, Vilela-Silva AC, Mourao PA. Biosynthesis of chondroitin sulfate: from the early, precursor discoveries to nowadays, genetics approaches. Adv Pharmacol 2006; 53: 117-140.

15. Warkentin TE, Greinacher A, Koster A, Lincoff AM. Treatment and prevention of heparin-induced thrombocytopenia: American College of Chest Physicians Evidence-Based Clinical Practice Guidelines (8th Edition). Chest 2008; 133: 340S-380S

16. Kawashima H, Atarashi K, Hirose M, Hirose J, Yamada
$\mathrm{S}$, Sugahara $\mathrm{K}$, et al. Oversulfated chondroitin/dermatan sulfates containing GlcAbeta1/ldoAalpha1-3GaINAc(4,6-Odisulfate) interact with L- and P-selectin and chemokines. J Biol Chem 2002; 277: 12921-12930.

17. Kawashima H, Hirose M, Hirose J, Nagakubo D, Plaas AH, Miyasaka M. Binding of a large chondroitin sulfate/dermatan sulfate proteoglycan, versican, to L-selectin, P-selectin, and CD44. J Biol Chem 2000; 275: 35448-35456.

18. Belmiro CL, Castelo-Branco MT, Melim LM, Schanaider A, Elia C, Madi K, et al. Unfractionated heparin and new heparin analogues from ascidians (chordate-tunicate) ameliorate colitis in rats. J Biol Chem 2009; 284: 11267-11278.

19. Dolber PC, Spach MS. Picrosirius red staining of cardiac muscle following phosphomolybdic acid treatment. Stain Technol 1987; 62: 23-26.

20. Dolber PC, Spach MS. Conventional and confocal fluorescence microscopy of collagen fibers in the heart. $J$ Histochem Cytochem 1993; 41: 465-469.

21. Stegemann $\mathrm{H}$, Stalder K. Determination of hydroxyproline. Clin Chim Acta 1967; 18: 267-273.

22. Border WA, Noble NA. Cytokines in kidney disease: the role of transforming growth factor-beta. Am J Kidney Dis 1993; 22: 105-113.

23. Kuschert GS, Coulin F, Power CA, Proudfoot AE, Hubbard RE, Hoogewerf AJ, et al. Glycosaminoglycans interact selectively with chemokines and modulate receptor binding and cellular responses. Biochemistry 1999; 38: 12959-12968.

24. Border WA, Noble NA, Yamamoto T, Harper JR, Yamaguchi $Y$, Pierschbacher MD, et al. Natural inhibitor of transforming growth factor-beta protects against scarring in experimental kidney disease. Nature 1992; 360: 361-364.

25. Watanabe J, Muranishi H, Yuasa H. Uptake mechanism of fractionated $\left[{ }^{3} \mathrm{H}\right]$ heparin in rat parenchymal hepatocytes in primary culture: effect of transport inhibitors on the uptake. Biol Pharm Bull 1993; 16: 497-500. 